\title{
U-slot patch antenna with low RCS based on a metaferrite substrate
}

\author{
Yujie Liu ${ }^{*}$, Philip Beal, Henry Giddens, and Yang Hao \\ Department of Electronic Engineering and Computer Science, Queen Mary University of London, London, UK
}

Received: 26 February 2019 / Accepted: 21 October 2019

\begin{abstract}
Metamaterial ferrites or metaferrites are artificial magnetic materials which mimic the properties of ferrites at a certain frequency operation. Antenna engineers are therefore able to design and create artificial substrates which replicate the electrical properties of ferrites without actually using any in the construction. This is advantageous as ferrites can offer performance improvements to microstrip antennas, such as size reduction and wideband impedance matching. In this paper, a metaferrite substrate designed by the use of a genetic algorithm is presented. The metaferrite was optimized in order to obtain the magnetic responses at $9 \mathrm{GHz}$, for its use as the substrate of a microstrip antenna. As an example, a U-slot patch antenna based on the metaferrite is demonstrated, which can achieve stable radiation and $14 \mathrm{~dB}$ radar cross section (RCS) reduction performance in the measurement.
\end{abstract}

Keywords: Ferrite metamaterial / metaferrite / genetic algorithm / RCS reduction / patch antenna

\section{Introduction}

Loading antennas with magnetic materials is well known technique that can enable engineers to design wideband antennas while maintaining a relatively low profile [1]. Many previous researchers have focused on loading antennas with ferrite materials in order to minimize the antenna dimensions and increase the radiation efficiency $[2,3]$. The antenna presented in [4] investigates the influence of the magnetic materials on the performance of dielectric resonator antenna (DRA) and it confirms that maintaining a certain ratio of $\mu_{r}$ to $\varepsilon_{r}$ is critically important to control the proximity of adjacent co-polar modes and create the DRA with a wide bandwidth of operation. However, naturally occurring magnetic materials may actually lose their magnetic properties up to microwave or millimeter-wave frequency, limiting their applications to higher frequencies. Therefore, many efforts have been spent over the years on the study of metamaterials, especially those with artificial magnetic properties [5]. Some studies have shown how metamaterials formed of dielectric substrates with periodic metallization can be designed to behave as artificial magnetic or magnetodielectric materials with required effective permittivity and permeability at a particular frequency [6]. For example, by using the spiral resonator

\footnotetext{
* e-mail: yujie.liu@qmul.ac.uk
}

[7] or metasolenoid [8] as "meta-atoms", an artificial magnetic material can be obtained with high permeability beyond microwave frequency which is easy to fabricate by low-cost photo-etching technology.

Many of these designs are achieved by the use of the Genetic Algorithm (GA) optimization procedure [9]. Furthermore, they can be also interpreted as materials having high impedance surfaces for antenna operation [10]. In [11], an approach is used to obtain required magnetic response at the specified operation frequency or frequencies by synthesizing the high impedance surface resistance and reactance. Since these two values are strongly related to the effective permeability of the equivalent material, therefore, it can be perceived as a valid approach to design "metamaterial ferrite" or "metaferrite" by optimizing the surface impedance of a bulk material. In addition when considering both permittivity and permeability, these kinds of artificial materials also have the performance of impedance matching, which can be utilized as absorbers without integrating any lossy elements, as presented in [12].

In this paper, a metaferrite material is designed and optimized to achieve a high effective permeability of 3 based on the GA technique at the working frequency of $9 \mathrm{GHz}$. A U-slot patch antenna positioned above this metaferrite substrate is proposed with the $14 \mathrm{~dB}$ RCS reduction at the normal incidence and its operation frequency band covers from $8.5 \mathrm{GHz}$ to $9.1 \mathrm{GHz}$ while $S_{11}<-9 \mathrm{~dB}$. 


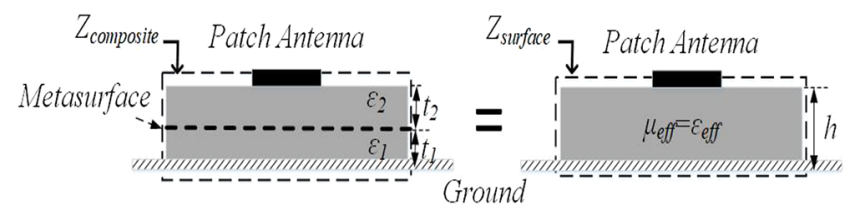

Fig. 1. Cross sectional diagram of the meteferrite patch antenna and its equivalent magnetic material with effective $\mu_{\text {eff }}=\varepsilon_{\text {eff }}$.

\section{Design procedure of the metaferrite material}

Figure 1 shows a diagram of the microstrip patch antenna positioned on the metaferrite substrate, which consists of two dielectric slabs enclosing a metasurface. The stack can be considered as a single substrate of material with equal effective permittivity and permeability, as described in [12]. The bottom layer has a thickness of $t_{1}$ and $\varepsilon_{1}$ permittivity and the upper layer has a thickness of $t_{2}$ and relative permittivity of $\varepsilon_{2}$. With the same PEC ground, the equivalent composite structure is a homogenous meteferrite material with the thickness of $h=t_{1}+t_{2}$.

As explained in [12], the surface impedance of the composite dielectric structure can be represented as $Z_{\text {composite. }}$ Since the surface impedance is given by [13],

$$
Z_{\text {surface }}=Z_{s} \tanh \left(j \omega \sqrt{\varepsilon_{0} \mu_{0}} n_{s} h\right),
$$

where $Z_{s}=Z_{0} \sqrt{\mu_{r} / \varepsilon_{r}}$, and $Z_{0}$ is the characteristic impedance of the vacuum and is equal to $377 \Omega$, by setting the $Z_{\text {composite }}$ equal to the surface impedance $Z_{\text {surface }}$ of the equivalent metaferrite material, the effective refractive index of the equivalent metaferrite substrate can be extracted and shown to be equal to

$$
\begin{gathered}
n_{s}=\mu_{r}=\varepsilon_{r}=\frac{1}{j \beta_{0} h} \tanh ^{-1}\left(\frac{Z_{\text {composite }}}{Z_{0}}\right) \\
Z_{\text {composite }}=Z_{0} \times \frac{1+S_{11}}{1-S_{11}}
\end{gathered}
$$

$Z_{\text {composite }}$ can be obtained by calculating the reflection coefficient $S_{11}$ through the equation (3). In this case, the effective value of $\mu_{\text {eff }}=\varepsilon_{\text {eff }}$ can be designed by modifying the surface impedance of the composite structure, by optimising the metasurface structure.

Here, in order to achieve the desired magnetic response of the composite substrate, the genetic algorithm (GA), a well-known nature-inspired global optimization technique, was applied to design this metasurface. As illustrated in the flowchart of the GA's process in Figure 2, the algorithm uses a combination of MATLAB code, which comprises the learning algorithm itself and commercial simulation software (CST microwave studio). The generated structure is built and analyzed through CST for calculating the $S_{11}$ parameters of the metaferrite unit cell, with periodic boundaries terminating the simulation space in the $x$ and $y$

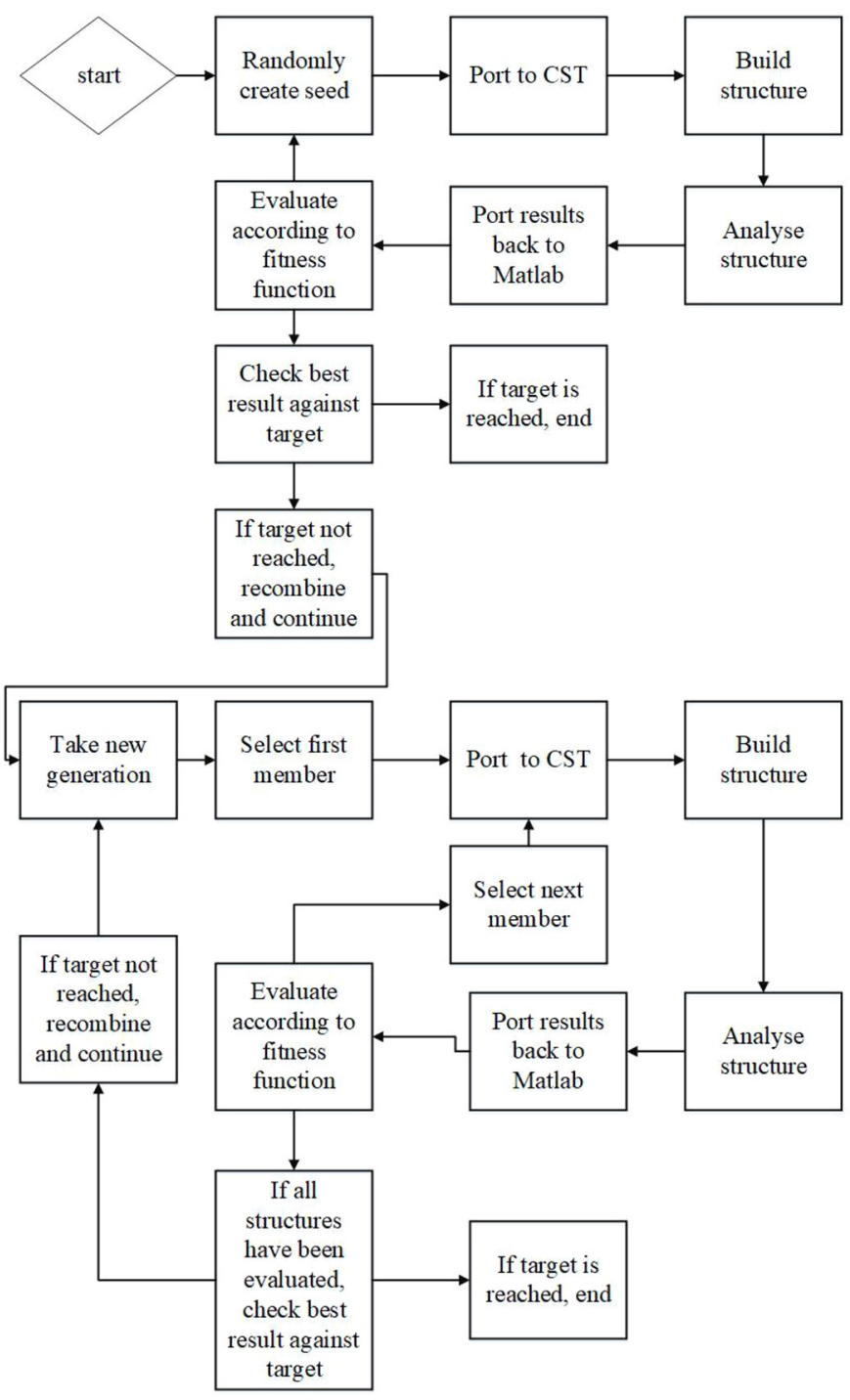

Fig. 2. Flow chart of the genetic algorithm optimization related to the CST studio for the metaferrite design.

directions. The results are passed back to the GA's fitness function for evaluating. Here the fitness function was:

$$
F F=-\left[\left(\mu_{r}^{\prime}(f)-3\right)^{2}+\left(\mu_{r}^{\prime \prime}(f)-1\right)\right]
$$

where $\mu_{r}^{\prime}$ and $\mu_{r}^{\prime \prime}$ are the real and imaginary values of the equivalent metaferrite material's effective permittivity, derived from its surface impedance $\left(Z_{\text {composite }}\right)$ by equations (2) and (3). The fitness function was evaluated at a frequency of $9 \mathrm{GHz}$.

The unit cell of the composite structure here is divided into $18 \times 18$ pixels with $1 \mathrm{~mm}$ intervals, such the metallized pixel would be encoded as 1 and the un-metallized pixel is encoded as 0 . Each pixel in the unit cell is represented by a single bit in the GA chromosome. In order to simplify the selection process, the program uses a 9-bit data string that is then extended to 45 cells via an L-system algorithm [14]. This forms the basis of the structure and provides a means 


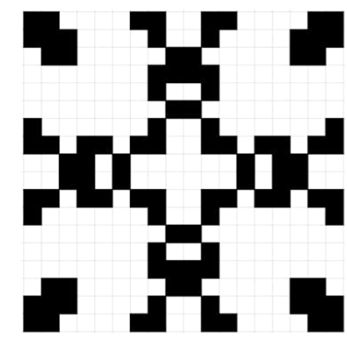

(a)

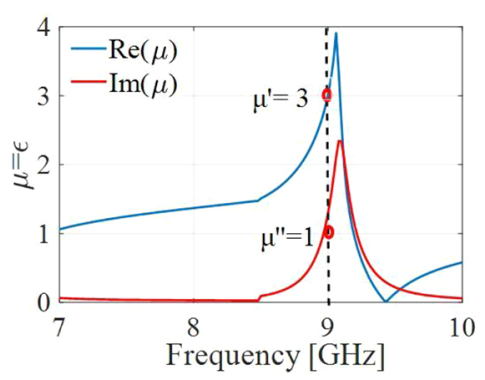

(b)
Fig. 3. (a) Optimised unit cell structure of the metaferrite surface. The black spots represent the metallized pixels which are encoded as 1, and the blank ones are unmetallized pixels which are encoded as 0. (b) Simulated real and imaginary components of $\mu_{\text {eff }}=\varepsilon_{\text {eff }}$ of finalised metaferrite substrate with $\varepsilon_{1}=4.3$, $t_{1}=0.8 \mathrm{~mm}, \varepsilon_{2}=6, t_{2}=1.52 \mathrm{~mm}$.

of exerting some control upon the final shape. The eightfold-symmetric pattern in the unit cell reduces the complexity of the design and ensures the structure has a polarization independent response, as it meant that a larger surface area could be created using only a small variable section and minimize the computational time.

The final optimized pattern of the unit cell for the metaferrite material design is shown in Figure 3a. This pattern can be easily fabricated by using the photoetching technology on the Rogers TMM6 substrate with the thickness of $t_{2}=1.52 \mathrm{~mm}$ and the permittivity of $\varepsilon_{2}=6$. By adding a $0.8 \mathrm{~mm}$ blank FR4 substrate with a conducted ground to the bottom, seen from Figure 1, the equivalent effective permeability and permittivity can be obtained through the methodology mentioned above.

Figure $3 \mathrm{~b}$ depicts the simulated real and imaginary component of the equivalent metaferrite material. It can be seen that this composite structure has the highest magnetic responses at about $9.1 \mathrm{GHz}$, and its corresponding effective permeability is 3.9 . The fitness function here was to achieve the metaferrite substrate with effective material properties of $\varepsilon_{r}=\mu_{r}=3$-j1 for the RCS reduction application at the frequency of $9 \mathrm{GHz}$, which was successfully achieved. This proposed composite structure can work as a frequency dependent metaferrite material during the frequency band from $8 \mathrm{GHz}$ to $9.2 \mathrm{GHz}$ since their effective permeability and permittivity are both above 1 .

\section{Metaferrite materials for RCS reduction of U-slot microstrip patch antenna}

The U-slot patch antenna is a well-known broadband microstrip antenna and has been studied to apply for the high frequency and high speed communication since proposed in 1995 by Huynh and Lee [15]. By adding this U-shaped slot to the rectangular patch, the currents on the patch surface are modified and different resonant modes are excited to achieve wideband performance. The design procedure has been presented in detail in [16]. Based on the metaferrite obtained above, we first design the U-slot patch antenna on the top of this effective magnetic substrate to gain the RCS reduction while maintain the stable radiation and low profile characteristics. The initial parameters of the U-shaped slot are calculated according to [16], but they still need to be optimized since the magnetic substrate here is frequency dependent. Figure 4a illustrates the $3 \mathrm{D}$ view of the proposed antenna and the U-shaped slot dimensions. The final optimized parameters for the impedance matching of this metaferrite based antenna are as follows: $\mathrm{W}=\mathrm{L}=180 \mathrm{~mm}, \quad \mathrm{Ws}=\mathrm{Ls}=33 \mathrm{~mm}, \quad \mathrm{St}=1.1 \mathrm{~mm}, \quad \mathrm{Lt}=$ $15.53, \mathrm{Wt}=7.76$. This antenna is coaxially fed and its feeding point is located in the center of the U-shaped slot. As seen from the same figure, the U-slot center is $1.82 \mathrm{~mm}$ away from the original point in the $-y$ direction. The photographs of the fabricated metasurface structure and the U-slot patch antenna are presented in Figure $4 \mathrm{~b}$, and they are both manufactured on the same substrate of Rogers TMM6 at the bottom and top sides, respectively. The total thickness of the antenna is the same with the thickness of the metaferrite material, which $h=t_{1}+t_{2}$ is $2.32 \mathrm{~mm}$

Figure 5 shows the comparison between the simulated and the measured results of the reflection coefficient $S_{11}$. The simulated $S_{11}$ obtained by the full-wave HFSS simulation is below $-9 \mathrm{~dB}$ from $8.5 \mathrm{GHz}$ to $9.1 \mathrm{GHz}$, which covers the same frequency band with the metaferrite material. It indicates that the proposed antenna is working well with the equivalent magnetic substrate. During the measurement, the resonant frequencies shift in the $S_{11}$ shift upwards, which is caused by the air gap in the practical antenna between the metasurface and the blank FR4 substrate (the air gap has an effect of lowering the effective material properties of the metaferrite). The radiation patterns at $8.65 \mathrm{GHz}, 8.8 \mathrm{GHz}$ and $9 \mathrm{GHz}$ can be seen in Figure 6. The differences between the simulated and measured are also likely due to manufacturing errors, as the air gap between those two substrates is inevitable in practical structure and also hard to quantify for the simulation.

In order to measure the RCS performance of the proposed antenna, a plane wave was launched from a standard horn antenna at the bottom onto the surface of the proposed antenna in the anechoic characterization chamber, as shown in Figure 7a. The RCS response can be extracted by subtracting the reflection coefficient of a same size PEC reflector positioned in the same place as the antenna. Time-gating was used to remove any further reflections from the horn antenna that were not accounted for during the calibration of the cables. In this case, the RCS responses of the metaferrite based U-slot patch antenna have been measured and compared with the simulated result. As illustrated in Figure 7b, the simulated result indicates that the antenna has a $-9 \mathrm{~dB}$ RCS reduction at around $9 \mathrm{GHz}$, and the measured RCS reduction is shifted to higher frequency of $9.35 \mathrm{GHz}$ due to the same reason of the air gap fabricated issue mentioned above. Besides that, the RCS reduction has been significantly increased while comparing to the same 

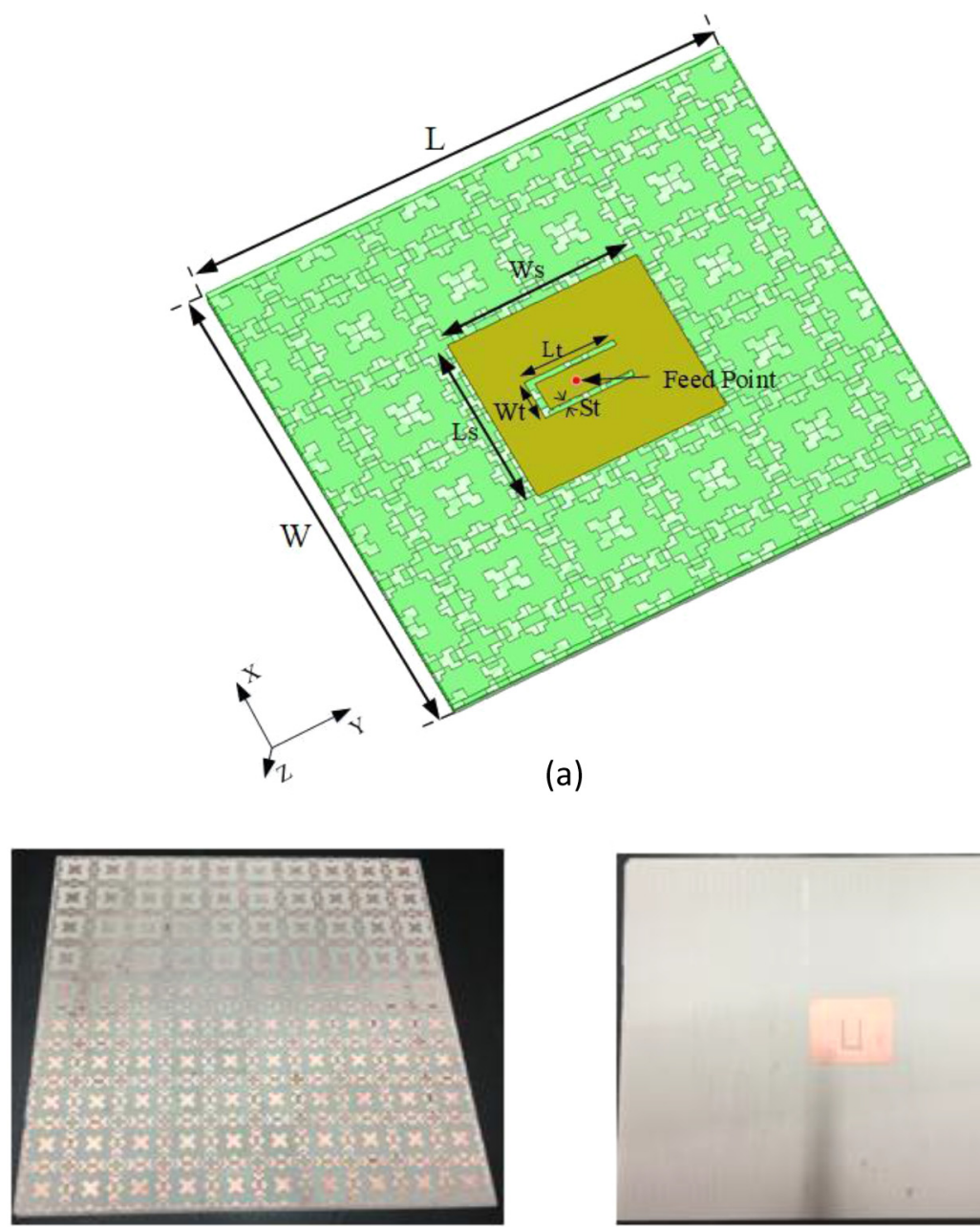

(b)

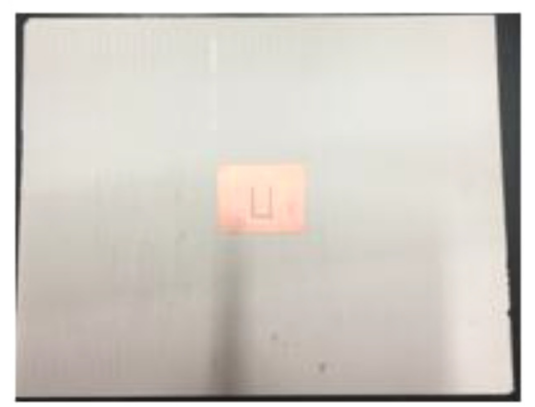

(c)

Fig. 4. (a) 3D view of the U-slot patch antenna based on the equivalent metaferrite substrate. The photograph of (b) the matesurface on the bottom of the Rogers TMM6 substrate, (c) U-slot patch antenna on the top.

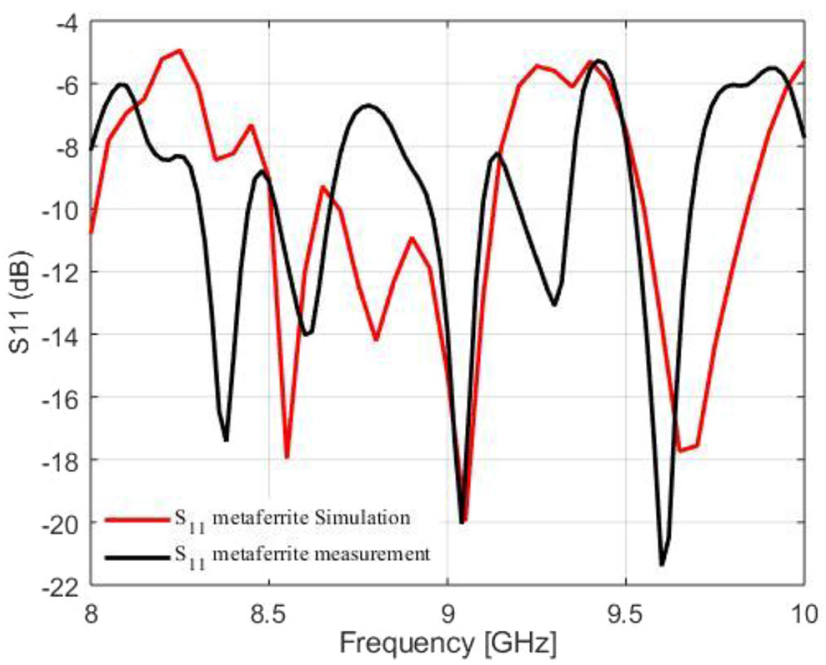

Fig. 5. Simulated and measured reflection coefficient $S_{11}$ of the U-slot patch antenna with metaferrite material. antenna without the optimized metasurface in between those two substrates, which means that the low RCS performance for the antenna can be obtained by applying the metaferrite substrate.

\section{Conclusion}

In this paper, we have discussed how the metaferrite can be utilized for antenna applications. The metaferrite was designed through genetic algorithm (GA) optimization. A U-slot patch antenna positioned on this effective magnetic substrate to achieve the additional RCS reduction performance while maintaining the wideband and stable radiation properties, which may be useful for military/ stealth applications when low profile antennas need to be positioned outward facing surfaces. Future optimized designs will focus on achieving a wide-band RCS reduction whilst maintaining good radiation performance of planar antennas. 


\section{E Plane}

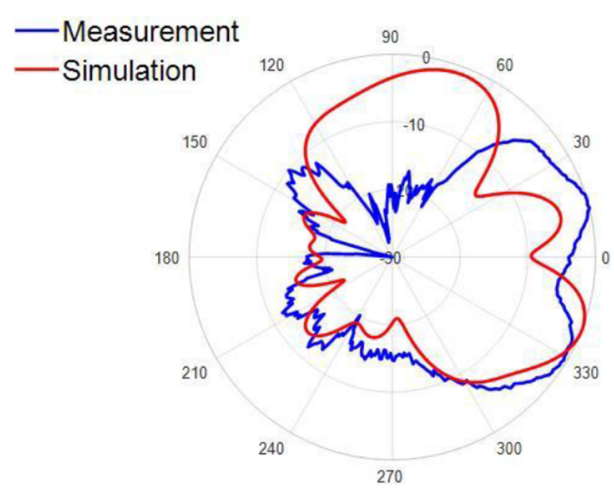

H Plane

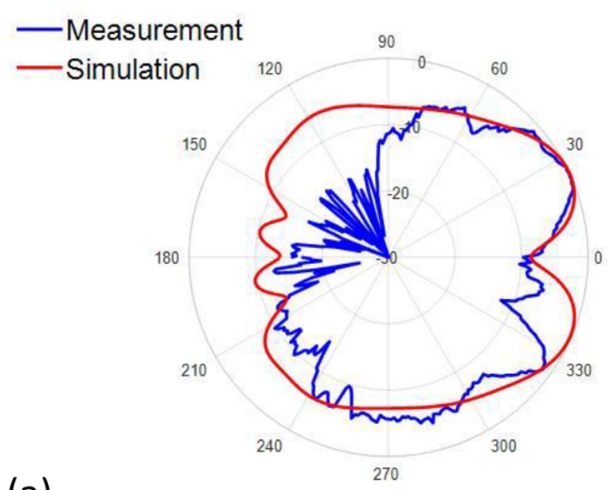

(a)

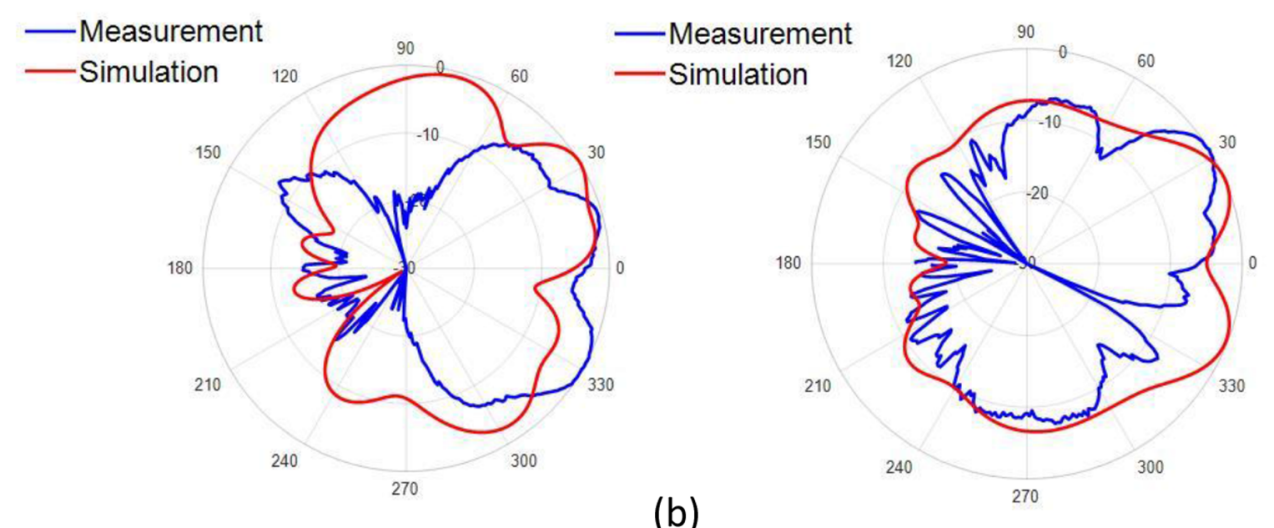

(b)

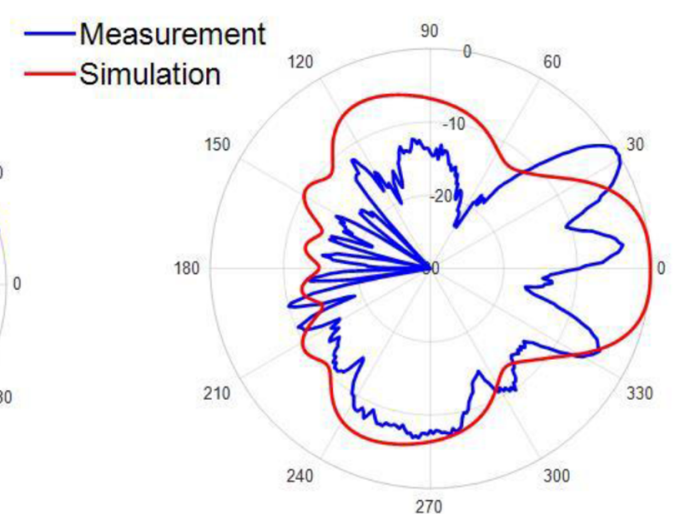

(c)

Fig. 6. Normalized measured and simulated radiation pattern of the metaferrite based U-slot patch antenna with E- and H-plane at the frequencies of (a) $8.65 \mathrm{GHz}$, (b) $8.8 \mathrm{GHz}$ and (c) $9 \mathrm{GHz}$. 


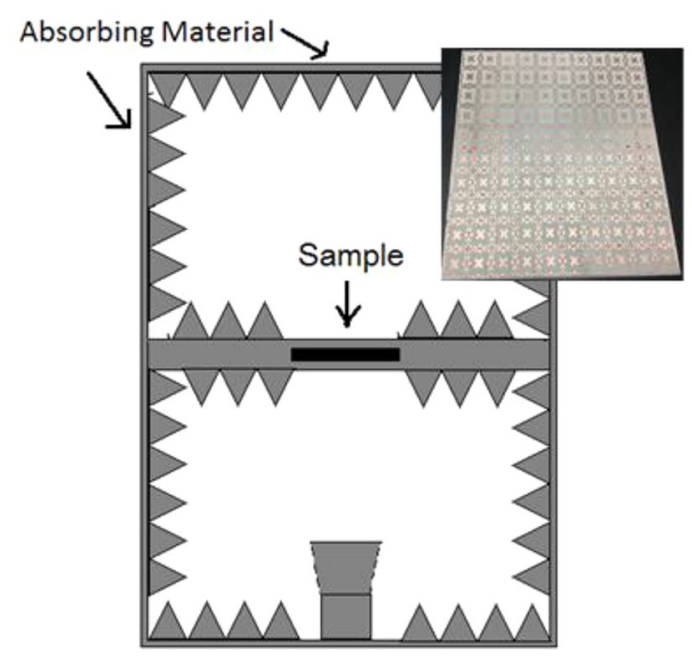

Horn Antenna

(a)

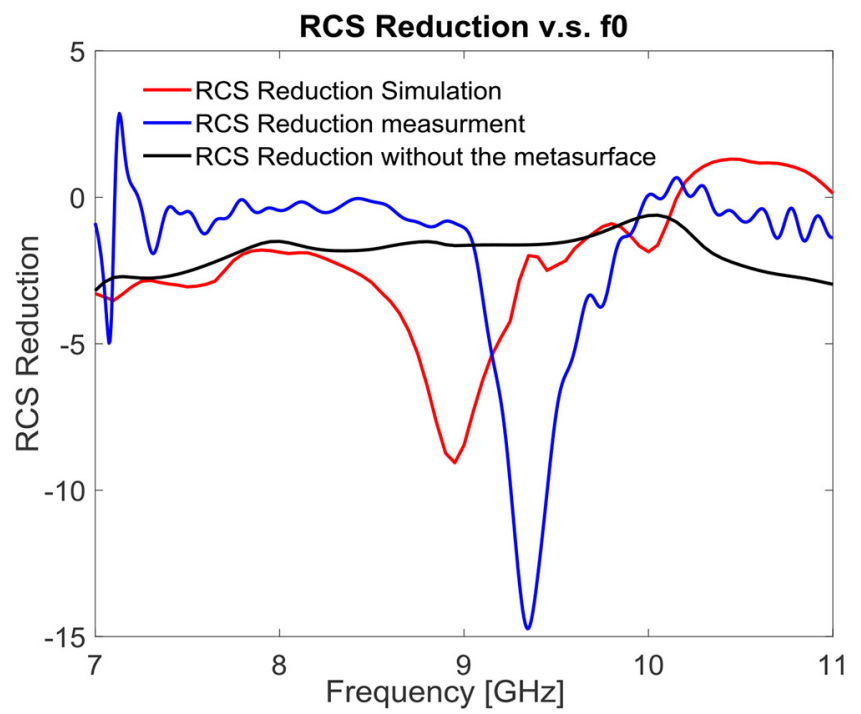

(b)

Fig. 7. (a) Diagram of the measurement setup and the fabricated metaferrite based U-slot patch antenna, (b) the Simulated and measured RCS reduction results of the metaferrite antenna and the comparison between the same patch antenna with the conventional dielectric substrate.

\section{References}

1. J.A. Kasemodel, Low-Profile Ferrite Loaded UWB Tightly Coupled Dipole Array, in IEEE Antennas and Propagation Society, AP-S International Symposium, 2011
2. J.R. James, A. Henderson, Electrically short monopole antennas with dielectric or ferrite coatings, Proc. Inst. Elect. Eng. 125, 793-803 (1978)

3. J.R. James, A. Henderson, Investigation of electrically small VHF and HF cavity-type antennas, in Proc. Inst. Elect. Eng. Conf. on An- tennas and Propagation, London, U.K., 1978, pp. $322-326$

4. J.R. James, R. Chair, K.M. Luk, K.M. Chow, K.W. Leung, J.C. Vardaxoglou, Influence of magnetic material on dielectric resonator antenna excitation, in Proc. Inst. Elect. Eng. Microw. Antennas and Propag., vol. 151, no. 4, pp. 293-298, 2004

5. H. Mossalaei, K. Sarabandi, Magneto-dielectrics in electromagnetics: concept and applications, IEEE Trans. Antennas Propag. 52, 1558-1567 (2004)

6. S. O'Brian, J.B. Pendry, Magnetic activity at infrared frequencies in structured photonic crystals, J. Phys: Condens. Matter 14, 6383-6394 (2002)

7. J.D. Baena, R. Marques, F. Medina, Artificial magnetic metamaterial design by using spiral resonators, Phys. Rev. B 69, 04402 (2004)

8. S. Maslovski, P. Ikonen, I. Kolmakov, S. Tretyakov, Artificial Magnetic Material Based on the New Magnetic Particle: Metasolenoid, in Progress In Electromagnetic Research, PIER 54, 61-81, 2005

9. L. Lanuzza, A. Monorchio, D.J. Kern, D.H. Werner, A robust GA-FSS technique for the synthesis of optimal multi-band AMC's with angular stability, in Proc. IEEE AP-S/URSI Symp. Dig., vol. 2, 2003, pp. 419-422

10. D. Sievcnpiper, L. Zhang, R.F. Limenez Broas, N.G. Aleropolous, E. Yablonovitch, High impedancc electromagnetic sufaces with a forbidden frequency band, IEEE Trans. Microwove Theory Technol. 47, 2059-2074 (1999)

11. D.J. Kern, D.H. Werner, M. Lisovich, Metaferrites: Using electromagnetic bandgap structures to synthesize metamaterial ferrites, IEEE Trans. Antennas Propag. 53, 1382-1389 (2005)

12. Z. Bayraktar, M.D. Gregory, X. Wang, D.H. Werner, Matched impedance thin planar composite magneto-dielectric metasurfaces, IEEE Trans. Antennas Propag. 60, 1910 1920 (2012)

13. K.J. Vinoy, R.M. Jha, Radar Absorbing Materials: From Theory to Design and Characterization (Kluwer, Boston, MA, 1996)

14. P. Prusinkiewicz, A. Lindenmayer, The Algorithmic Beauty of Plants, Refina, Saskatchewan, Canada, 1996, pp. $28-46$

15. T. Huynh, K.F. Lee, Single-layer single-patch wideband microstrip antenna, Electr. Lett. 31, 1310-1312 (1995)

16. S. Weigand, G.H. Huff, K.H. Pan, J.T. Bernhard, Analysis and design of broad-band single-layer rectangular U-slot microstrip patch antennas, IEEE Trans. Antennas Propag. 51, 457-468 (2003)

Cite this article as: Yujie Liu, Philip Beal, Henry Giddens, Yang Hao, U-slot patch antenna with low RCS based on a metaferrite substrate, EPJ Appl. Metamat. 6, 20 (2019) 